neuropsychiatrie

vereinigt mit psychiatrie \& psychotherapie

\title{
Bericht des Präsidenten
}

\author{
Paulus Hochgatterer · Anna-Katharina Purtscher-Penz · Andreas Karwautz
}

Online publiziert: 11 . Juni 2014

(C) Springer-Verlag Wien 2014

\section{Liebe Kolleginnen und Kollegen,}

am Ende meiner Periode als Präsident sei es mir erlaubt, im Sinn einer kurzen Bilanz die wichtigsten Dinge anzuführen, die von Seiten der ÖGKJP in den vergangenen zweieinhalb Jahren für unser Fach erreicht bzw. initiiert wurden.

Im Bereich der Aus- und Weiterbildung konnte nach erfolgreicher Pilotphase die ,Werkstatt Gösing ' als ein praxisnahes Weiterbildungsformat für Fachärztinnen und Fachärzte unserer Sparte fix installiert werden. Die Arbeiten an der Neuformulierung des Rasterzeugnisses im Rahmen der kommenden Revision der Ärzteausbildungsordnung sind weit gediehen. Unter anderem werden für den letzten Teil der Ausbildung gemeinsam mit der ÖGKJ und der

Prim. Dr. P. Hochgatterer $(\bowtie)$

Landesklinikum Tulln,

Alter Ziegelweg 10,

3430 Tulln, Österreich

E-Mail: paulus.hochgatterer@tulln.lknoe.at

Prima. Drin. A.-K. Purtscher-Penz

Neuropsychiatrie Kinder- und

Jugendabteilung, LSF Graz,

Wagner Jauregg Platz 1 ,

8036 Graz, Österreich

E-Mail: katharina.purtscher@lsf-graz.at

Univ.-Prof. Dr. A. Karwautz

Universitätsklinik für Kinder- und

Jugendpsychiatrie, Medizinische

Universität Wien,

Währinger Gürtel 18-20,

1090 Wien, Österreich

E-Mail: andreas.karwautz@meduniwien.ac.at
ÖGPP Module geplant, die jeweils von beiden Fachausbildungen zugänglich sein sollen.

Die aufwändige Formulierung und letztlich Etablierung der Leitlinien zur Off-Label-Behandlung in der Kinderund Jugendpsychiatrie schafft nicht nur Sicherheit in einer hoch sensiblen Zone unseres Faches, sondern ist insgesamt als äußerst wichtige Maßnahme der Qualitätssicherung nicht hoch genug einzuschätzen.

Die Anerkennung als Mangelfach mit der daraus folgenden Einberechnung des jeweiligen Abteilungsleiters für die Zahl der anzuerkennenden Ausbildungsstellen hat sich als unzureichende Maßnahme zur raschen Erhöhung der Zahl an Fachärztinnen und Fachärzten herausgestellt. Daher wurde eine Initiative in Richtung Ärztekammer und Gesundheitsministerium gestartet, den üblichen Ausbildungsschlüssel von 1:1 auf $1: 2$ (pro Fachärztin/Facharzt zwei Ausbildungsärztinnen/Ausbildungsärzte) zu verbessern.

Im Bereich des LKF-Systems gelang es, den Berechnungsmodus in unserem Fach in Richtung Tagesbepunktung zu verändern. Dies hat dazu geführt, dass die an unseren Spitalsabteilungen üblichen längeren Aufenthaltsdauern in adäquater Weise honoriert werden. Zusätzlich war es möglich, deutlich verbesserte und zugleich verbindliche Personalausstattungskriterien in das System aufnehmen zu lassen.

Zuletzt wurde mit dem Rückhalt der breiten Zustimmung unserer Mitglieder das Projekt der Umbenennung unseres Faches in ,Kinder- und Jugendpsychia- trie und Psychotherapeutische Medizin' (das „zweite P“) in Form eines entsprechenden Ansuchens an die Österreichische Ärztekammer konkret in Angriff genommen. Damit soll dem Faktum, dass Psychotherapie in der Österreichischen Kinder- und Jugendpsychiatrie seit jeher ein zentrales Behandlungsprinzip darstellt, entsprechend Rechnung getragen werden.

Zuletzt gelang es, den Kongress der ESCAP (European Society for Childand Adolescent Psychiatry) für das Jahr 2019 nach Wien zu holen. Damit wird seit längerem wieder eine große internationale Tagung unseres Faches in Österreich stattfinden.

Schließlich sei auch hier noch einmal betont, dass sich die Installierung der neuropsychiatrie als offizielles wissenschaftliches Organ unserer Fachgesellschaft bereits in den ersten eineinhalb Jahren außerordentlich bewährt hat.

Ich darf mich bei meinen Kolleginnen und Kollegen im Vorstand der ÖGKJP für das große Wohlwollen, für die hohe Bereitschaft sowohl zur gegenseitigen Unterstützung als auch zur Eigeninitiative und für die ausgesprochen angenehme Atmosphäre in den Sitzungen ganz herzlich bedanken. Andreas Karwautz, meinem Nachfolger, wünsche ich das Allerbeste, vor allem ein ähnliches Vergnügen in der Begleitung der Entwicklung unseres Faches, wie ich es haben durfte.

Mit besten Grüßen,

Prim. Dr. P. Hochgatterer

Präsident der ÖGKJP (2012-2014) 